\title{
Three dimensional HiLo-based structured illumination for a digital scanned laser sheet microscopy (DSLM) in thick tissue imaging
}

\author{
Dipanjan Bhattacharya, ${ }^{1,2,6^{*}}$ Vijay Raj Singh, ${ }^{1}$ Chen Zhi, ${ }^{1,3}$ Peter T. C. So, ${ }^{1,4,5}$ Paul \\ Matsudaira, ${ }^{1,2,6}$ and George Barbastathis ${ }^{1,4}$ \\ ${ }^{1}$ Singapore-MIT Alliance for Research and Technology (SMART) Centre, 117543, Singapore \\ ${ }^{2}$ Centre for BioImaging Science (CBIS), National University of Singapore, 117546, Singapore \\ ${ }^{3}$ Department of Bioengineering, National University of Singapore, 117576, Singapore \\ ${ }^{4}$ Department of Mechanical Engineering, Massachusetts Institute of Technology, Cambridge, MA, 02139, USA \\ ${ }^{5}$ Department of Biomedical Engineering, Massachusetts Institute of Technology, Cambridge, MA, 02139, USA \\ ${ }^{6}$ MechanoBiology Institute-Singapore and Department of Biological Sciences, National University of Singapore, \\ 117411, Singapore \\ *dipanjan@smart.mit.edu
}

\begin{abstract}
Laser sheet based microscopy has become widely accepted as an effective active illumination method for real time three-dimensional (3D) imaging of biological tissue samples. The light sheet geometry, where the camera is oriented perpendicular to the sheet itself, provides an effective method of eliminating some of the scattered light and minimizing the sample exposure to radiation. However, residual background noise still remains, limiting the contrast and visibility of potentially interesting features in the samples. In this article, we investigate additional structuring of the illumination for improved background rejection, and propose a new technique, "3D HiLo" where we combine two HiLo images processed from orthogonal directions to improve the condition of the $3 \mathrm{D}$ reconstruction. We present a comparative study of conventional structured illumination based demodulation methods, namely 3Phase and HiLo with a newly implemented 3D HiLo approach and demonstrate that the latter yields superior signal-tobackground ratio in both lateral and axial dimensions, while simultaneously suppressing image processing artifacts.
\end{abstract}

(C)2012 Optical Society of America

OCIS codes: (180.6900) Three-dimensional microscopy; (180.2520) Fluorescence microscopy; (110.6880) Three-dimensional image acquisition; (100.3020) Image reconstruction-restoration.

\section{References and links}

1. C. J. Sheppard and T. Wilson, "The theory of the direct-view confocal microscope," J. Microsc. 124(2), 107-117 (1981).

2. J. B. Pawley, Handbook of Confocal Microscopy (Plenum: New York, 1995).

3. O. E. Olarte, J. Licea-Rodriguez, J. A. Palero, E. J. Gualda, D. Artigas, J. Mayer, J. Swoger, J. Sharpe, I. RochaMendoza, R. Rangel-Rojo, and P. Loza-Alvarez, "Image formation by linear and nonlinear digital scanned lightsheet fluorescence microscopy with Gaussian and Bessel beam profiles," Biomed. Opt. Express 3(7), 1492-1505 (2012).

4. A. H. Voie, D. H. Burns, and F. A. Spelman, "Orthogonal-plane fluorescence optical sectioning: threedimensional imaging of macroscopic biological specimens," J. Microsc. 170(3), 229-236 (1993).

5. D. McLachlan, Jr., "Extreme focal depth in microscopy," Appl. Opt. 3(9), 1009 (1964).

6. R. R. Schram, "Light-scanning photomicrography," Microscope 29, 13-17 (1981).

7. P. J. Keller, A. D. Schmidt, J. Wittbrodt, and E. H. K. Stelzer, "Reconstruction of zebrafish early embryonic development by scanned light sheet microscopy," Science 322(5904), 1065-1069 (2008).

8. P. J. Keller, A. D. Schmidt, A. Santella, K. Khairy, Z. Bao, J. Wittbrodt, and E. H. K. Stelzer, "Fast, highcontrast imaging of animal development with scanned light sheet-based structured-illumination microscopy," Nat. Methods 7(8), 637-642 (2010).

9. G. Best, R. Amberger, D. Baddeley, T. Ach, S. Dithmar, R. Heintzmann, and C. Cremer, "Structured illumination microscopy of autofluorescent aggregations in human tissue," Micron 42(4), 330-335 (2011). 
10. L. Gao, N. Bedard, N. Hagen, R. T. Kester, and T. S. Tkaczyk, "Depth-resolved image mapping spectrometer (IMS) with structured illumination," Opt. Express 19(18), 17439-17452 (2011).

11. D. Karadaglić and T. Wilson, "Image formation in structured illumination wide-field fluorescence microscopy," Micron 39(7), 808-818 (2008).

12. J. Mertz, "Optical sectioning microscopy with planar or structured illumination," Nat. Methods 8(10), 811-819 (2011).

13. M. A. Neil, R. Juskaitis, and T. Wilson, "Method of obtaining optical sectioning by using structured light in a conventional microscope," Opt. Lett. 22(24), 1905-1907 (1997).

$<\mathrm{jrn}>14$. M. A. A. Neil, A. Squire, R. Juskaitis, R. Juskaitis, P. I. Bastiaens, and T. Wilson. "Wide-field optically sectioning fluorescence microscopy with laser illumination," J. Microsc.-Oxford 197, 1-4 (2000).</jrn>

15. T. Wilson, "Optical sectioning in fluorescence microscopy," J. Microsc. 242(2), 111-116 (2011).

16. J. Mertz and J. Kim, "Scanning light-sheet microscopy in the whole mouse brain with HiLo background rejection," J. Biomed. Opt. 15(1), 016027 (2010).

17. T. J. Schröter, S. B. Johnson, K. John, and P. A. Santi, "Scanning thin-sheet laser imaging microscopy (sTSLIM) with structured illumination and HiLo background rejection," Biomed. Opt. Express 3(1), 170-177 (2012).

\section{Introduction}

Three-dimensional (3D) thick tissue imaging in real time is an invaluable tool for visualizing directly the mechanisms of different biological processes in developmental, immunological, or other contexts. However, in vivo or in situ imaging with single cell resolution remains a major challenge. Existing techniques are based on variants of confocal microscopy [1,2] and can provide good lateral and moderate axial resolution, but they are limited by high photobleaching and poor temporal resolution. Confocal techniques are based on scanning in $3 \mathrm{D}$, so for large specimens (size $\sim 1 \mathrm{~mm}^{3}$ ), they become impractical. For the same reason, photobleaching is severe as the excitation occurs along the entire optical axis with variable beam waist as the sample is being scanned.

Recently, laser sheet based microscopic techniques [3-8] have emerged as a useful alternative for imaging biological tissue specimens of size larger than $\sim 1 \mathrm{~mm}^{3}$ and over long time periods with high speed, good resolution, and significantly reduced photo bleaching and phototoxic effects. Unlike confocal techniques, which use spot-like excitation, laser-sheet based methods attempt to excite an entire plane within the tissue sample; the illuminated plane is either formed at once using cylindrical lens (called selective plane illumination microscopy: SPIM) or by fast-scanning a pencil-like beam (called digital scan laser sheet microscopy: DSLM). The camera is oriented perpendicular to the illuminated plane, thus significantly speeding up the image acquisition time and, for the same reason, mitigating photobleaching. However, the propagation of a "plane" of light through large-thick tissue results in scattering artifacts in the final image.

Background "haze" is common even in conventional wide-field microscopy and structured illumination-based methods have been developed to reject it, with the aim of visualizing more clearly the in-focus fluorescent-plane $[9,10]$. By projecting a single spatial frequency grid pattern onto the sample, the microscope effectively images only parts of the grid that lie within its focal range, whereas the background is rejected by frequency mixing of in-focus information (in time-domain radar signal processing, similar approaches are often referred to as "phase locking"). Two approaches for background "haze" removal by demodulation have been proven to be particularly popular: the "3Phase" method $[8,11,15]$ and the "HiLo" method $[12,13]$.

The first method is based on recording images corresponding to the three different phases of the projected grid $\left(0^{\circ}, 120^{\circ}\right.$ and $240^{\circ}$ Phase shifts $)$ and was proposed first by Wilson and associates [11]. More recently, Mertz and associates presented a new approach [16], which requires only one uniform and one structured image at each imaging plane for the reconstruction of optical sectioned image. The final reconstructed image combines two images: the in-focus high frequency content (called "Hi" image) extracted from a uniformly illuminated image; and the in-focus low frequency content (called "Lo" image) obtained under structured illumination. The linear combination of "Hi" and "Lo" images with a scaling factor ' $\eta$ ' $(\mathrm{Hi}+\eta \mathrm{Lo})$ is used to ensure a seamless transition between spatial frequencies of the 
object features; for that reason the final post-processed image is called the "HiLo," from which term the technique derives its name as well.

Structured illumination methods have been combined with laser sheet microscopy recently for the suppression of background noise $[8,16,17]$ but they were applied mainly to imaging of relatively thin and optically clean biological samples. Here we present a structured illumination based version of DSLM that is successful in imaging thick tissue samples and compare the new technique quantitatively with existing structured illumination-based methods (3Phase and HiLo). We refer to our new approach as "3D HiLo," because it combines HiLo images from two orthogonal directions (excitation objective point of view and detection objective point of view) to reconstruct a 3D tissue image with subtracted background noise and low image processing artifacts.

The experimental apparatus for implementing conventional HiLo, 3D HiLo, and alternative structured illumination programs (such as 3Phase) is described in Section 2. In section 3, we describe the post-processing algorithms that we have devised to combine the HiLo images from two orthogonal directions. In section 4, we present numerical and experimental results of images acquired with these three alternate structured illumination programs and post-processing algorithms, and compare them quantitatively in terms of background rejection and image artifacts. Conclusions and some suggestions for follow-up work are presented in Section-5.

\section{Digital scanned light sheet microscope with multiple programs of structured illumination}

We modified the design of Digital Scanned Light-sheet Microscope (DSLM) [3] as shown in Fig. 1 to provide real time structured illumination to the sample according to the new 3D HiLo principle as well as older, alternative programs of structured illumination [4-8]. In our experimental apparatus, the illumination is provided by four laser lines: $405 \mathrm{~nm}$ (Coherent CUBE $403 \mathrm{~nm}$ ), $488 \mathrm{~nm}$ (Coherent Sapphire $488 \mathrm{~nm}$ ), $561 \mathrm{~nm}$ (Coherent Sapphire $561 \mathrm{~nm}$ ), and $659 \mathrm{~nm}$ (Coherent CUBE $659 \mathrm{~nm}$ ) so that we can excite different biological flurophores at will. All four laser lines are combined and passed through an excitation filter-wheel (Sutter instruments, Lambda 10-3) for excitation of biological samples, one color at a time. The selected laser beam then passes through a sequence of optics designed to produce the two orthogonal structured illumination images as follows: The first element, an acousto-optictunable filter, AOTF (AA optoelectronics, AOTFTnc-400-650-TN) adjusts the laser illumination power at any given wavelength, and also modulates the pencil beam sinusoidally to structure the illuminated "sheet." After the AOTF, the beam goes through a beam expander (1.5X, Edmund optics) which fills the back aperture of the objective, and a 2 axis mechanical optical scanner (Cambridge Technology, MicroMax 673). Finally, the beam goes through the excitation objective (Olympus 4X, 0.13 NA in air). Depending on how we program the AOTF and scanner, we can obtain an almost arbitrary structured (modulated) laser-sheet at the focal point of the collection objective (Zeiss 10X, 0.3 NA water immersion).

\#176294 - \$15.00 USD Received 14 Sep 2012; revised 25 Oct 2012; accepted 26 Oct 2012; published 20 Nov 2012

(C) 2012 OSA

3 December 2012 / Vol. 20, No. 25 / OPTICS EXPRESS 27339 


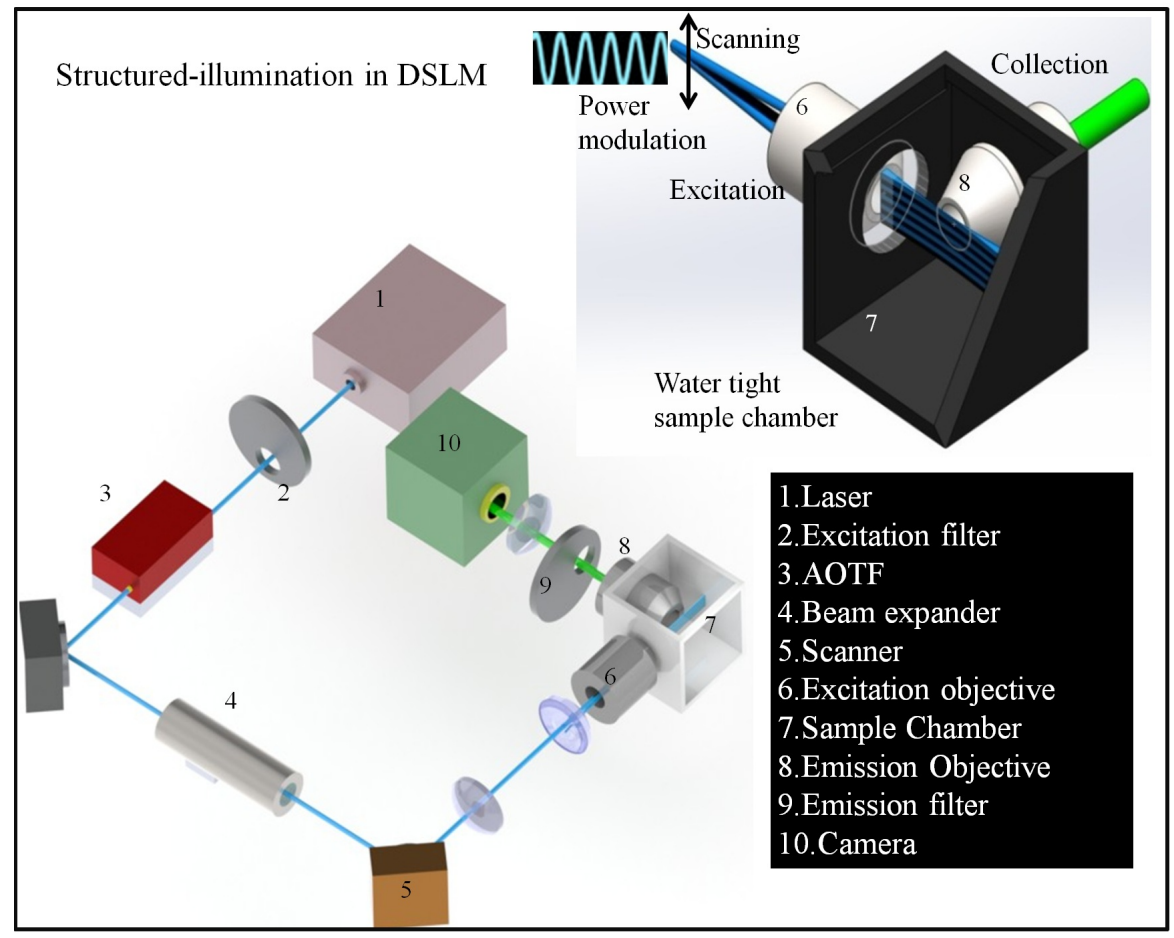

Fig.1: Experimental schematics of digital laser sheet microscopy using structured illumination for simultaneous acquisition of HiLo and 3Phase methodologies.

The sample is mounted from the top onto a tightly sealed water chamber, on a 4-axis stage (X-Y-Z- $\theta$ ) (Physik Instrumente, E-665, M-11k028, M-116DGH). The collection objective is inserted into the same sample chamber. We also used two piezo-stages (Physik Instrumente, P721.CLQ) for precise alignment of the illumination and collection objectives. The emission from the sample after the collection objective is passed through an electronically controlled emission filter-wheel and finally transduced by an Andor Clara camera. The whole system is computer controlled through Labview-2011 (National Instruments) using NI-DAQ SCB-68.

The combination of AOTF plus scanner allows us to implement different modes of structured illumination. A digitally controlled trigger signal is used to synchronize AOTF, scanner and camera so as to precisely reproduce the imprinted sinusoidal excitation pattern over different fluorescent planes, depending on the desired mode of structuring the illumination. For the 3D HiLo program, we capture two raw images. The first is a standard DSLM image where the pencil is scanned across the sample without any modulation. For the second raw image, we modulate the pencil so that the resulting three-dimensional (3D) structured illumination pattern from the combination of AOTF action and mechanical scanning consists of sheets oriented parallel to the XZ plane, as shown in Fig. 2. The two collected images can either be processed individually at each XY plane to yield slice-wise 3D images of the object according to conventional HiLo; or the additional structure along the YZ plane can be exploited to yield even better background rejection, as we describe in detail in section 3 .

Alternatively, to implement 3Phase, we expose the sample to sinusoidal patterns of light shifted by 0,120 , and 240 degrees in three consecutive laser scans on the plane of excitation $\mathrm{XY}$; these three collected fluorescent raw images are then post-processed according to the 3Phase scheme $[4,7]$ to reject the background. Due to the frame transfer rate of our camera, we are limited to capturing $\sim 4$ images ( 3 modes of structured illumination and one corresponding to uniform illumination) for each fluorescent plane in a time of less than 1 
second. We wish to emphasize that we are camera limited, and not signal limited; based on the amount of fluorescence collected from the sample, we estimate that by using a low-noise CMOS camera such as Hamamatsu-Orca Flash-4, or Andor Neo s-CMOS, we could image more than 10 times faster.

We imaged the fluorescently-labeled membrane and nucleus of live zebrafish at 6 and 36 hours post fertilization (hpf) to demonstrate and quantitatively compare 3Phase, conventional HiLo, and 3D HiLo. We microinjected mRNA inside freshly laid zebrafish embryo (1ng) using an Eppendorf microinjector. Cell nuclei were labeled by expression H2B-mRFP (or H2B-eGFP, depending upon experiments) while membranes were labeled by expression of a membrane bound pCAG-mGFP.

\section{Background removal in 3D HiLo}

The combined action of AOTF and mechanical scanning in our experiment creates rich spatial structure on the sample, which may be exploited in several ways. For example, if one looks at a single slice parallel to the $\mathrm{XY}$ plane at a fixed axial position $z$, there is a sinusoidal illumination pattern $\cos (k y)$ imposed, where $k$ is the spatial frequency. This raw structured illuminated image can be combined with the unstructured (uniformly illuminated) raw image of the same slice according to conventional HiLo to reject the background noise from this slice only. All the slices collected from different $z$ locations can then be put together to create a 3D post-processed image $I_{\mathrm{XY}}(x, y ; z)$, which we will refer to as XY-HiLo. (This is, of course, identical to conventional HiLo as described by Ref-12,13.)

Alternatively, if one looks at a single slice parallel to the $\mathrm{YZ}$ plane at a fixed lateral position $x$, there is again a sinusoidal pattern $\cos (k y)$ which can similarly combined with the unstructured raw image according to conventional HiLo. Thus, we can create an alternate a 3D post-processed image $I_{\mathrm{YZ}}(y, z ; x)$, which we will refer to as YZ-HiLo.

Even better, one can exploit the 3D structure of the modulated illumination to reject background more effectively than conventional HiLo with least artifacts by combining the XY and YZ versions. The combination is what we refer to as "3D HiLo." In this paper, we limit our analysis to linear convex combinations of the $I_{\mathrm{XY}}$ and $I_{\mathrm{YZ}}$ images according to,

$$
I_{3 D}(x, y, z)=\alpha(x) I_{\mathrm{XY}}(x, y ; z)+\{1-\alpha(x)\} I_{\mathrm{YZ}}(y, z ; x) .
$$

Where, the spatially variant mixing factor $\alpha(x)$ allows us to emphasize or de-emphasize the HiLo orientation that is most reliable at each spatial location. We have devised at least three methods for doing so:

(1) Mean method: As the name suggests, this method simply uses $\alpha(x)=1 / 2$, i.e. it takes the mean of the 3D-images computed by XY-HiLo and YZ-HiLo. Thus all the features present in either of the XY or YZ HiLo images are guaranteed to be retrieved; however, at the same time, the regions where the background has been well rejected in either one of those images but not the other can be compromised.

(2) Geometrically weighted mean method: Since we expect the image quality to deteriorate toward the direction away from the illumination objective in the XY-HiLo case, we can deemphasize the contribution of the corresponding HiLo orientations to the final 3D HiLo processed image. Some deterioration may also occur toward the direction away from the collection objective in the YZ-HiLo case. However, the second effect is negligible assuming the lateral size of the sample is much larger than its thickness. These expectations were confirmed by our experimental observations, as we report in the section 4 . We can accomplish this with a proper choice of the spatially variant mixing factor $\alpha(x)$. As simplest choice, in the experiments reported later we used

\#176294 - \$15.00 USD Received 14 Sep 2012; revised 25 Oct 2012; accepted 26 Oct 2012; published 20 Nov 2012

(C) 2012 OSA

3 December 2012 / Vol. 20, No. 25 / OPTICS EXPRESS 27341 


$$
\alpha(x)=\frac{x}{x_{\max }} .
$$

Where, $x_{\max }$ is the size of the sample; other choices such as exponential may be more physically justifiable, but we found that the choice has no significant impact on the result. A better alternative is proposed in method (3) below (Variance weighted.)

The disadvantage of the geometrically weighted mean method is that the choice of mixing factor $\alpha(x)$ is fixed a priori, without any opportunity to take into account the nature of the sample. For example, if the contrast of projected grid is poorer toward the illumination objective due to the structure of a particular highly inhomogeneous sample, this method will turn out to do exactly the opposite of what it should. This event is relatively rare, however, and can be further mitigated by judicious placement of the specimen in the sample holder.

(3) Variance weighted method: To combat the potential problem with inhomogeneous samples mentioned just before, we may define the mixing factor to depend on the local image fluctuations rather than the geometry directly. One may think of this as ad hoc adaptive weighting: we give large weight to portions of the image with large variation (rich structure) and penalize those with small variation, assuming that the latter is an artifact of the structured illumination locally having limited contrast. The simplest way to implement variance weighting is to select the mixing factor as

$$
\alpha(x)=\frac{\sigma_{\mathrm{XY}}(x)}{\sigma_{\mathrm{XY}}(x)+\sigma_{\mathrm{YZ}}(x)} .
$$

where $\sigma(x)$ is a locally computed estimate of the image standard deviation for the XY-HiLo and YZ-HiLo images, respectively. In our numerical implementation, we used a $15 \times 15$ pixel size window.

The relative performance of the three methods, as well as comparisons with conventional DSLM, conventional 3Phase, and conventional HiLo are reported in section 4 below. It should be noted that nonlinear methods for combining the XY-HiLo and YZ-HiLo raw images, such as expectation maximization, may also be used, but they are outside the scope of the present paper.

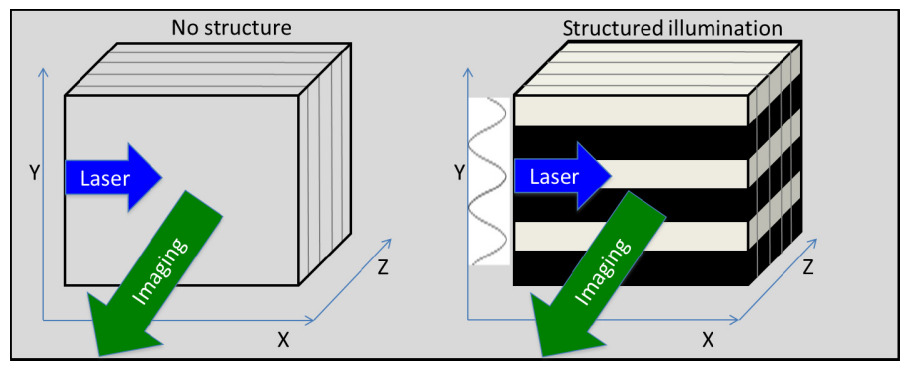

Fig. 2: Directional view of lateral XY-HiLo (lateral) and YZ-HiLo (axial).

\section{Results}

To model and compare the performance of the different flavors of 3D HiLo presented in section 3, we conducted simulations and experiments image formation for a collection of fluorescence beads. In the simulation, the beads were assumed to be $12 \mu \mathrm{m}$ and distributed in a $60 \mu \mathrm{m} \times 60 \mu \mathrm{m} \times 150 \mu \mathrm{m} 3 \mathrm{D}$ volume segmented into $150 \times 150 \times 150$ voxels. We assumed laser excitation wavelength $480 \mathrm{~nm}$ and fluorescence emission wavelength $530 \mathrm{~nm}$. Uniform and structured excitation patterns were simulated for the laser sheet, with thickness $5 \mu \mathrm{m}$, 
corresponding to the 150 axial planes. For structured illumination, the resulting spatial frequency of the sinusoidal structured pattern at the object plane was 392 lines 392 392 line pairs $/ \mathrm{mm}$. A microscope objective (water immersion) with NA 1.0 was used for collection of the fluorescence signal. The PSF of the raw (unprocessed) fluorescence image was computed assuming diffraction-limited optics. Quantitative analysis of reconstructed images was performed by plotting the normalized intensity cross-sections along the $\mathrm{x}-, \mathrm{y}-$ and $\mathrm{z}$-axes respectively to test the proposed method, and the results were compared with conventional DSLM (unstructured illumination).

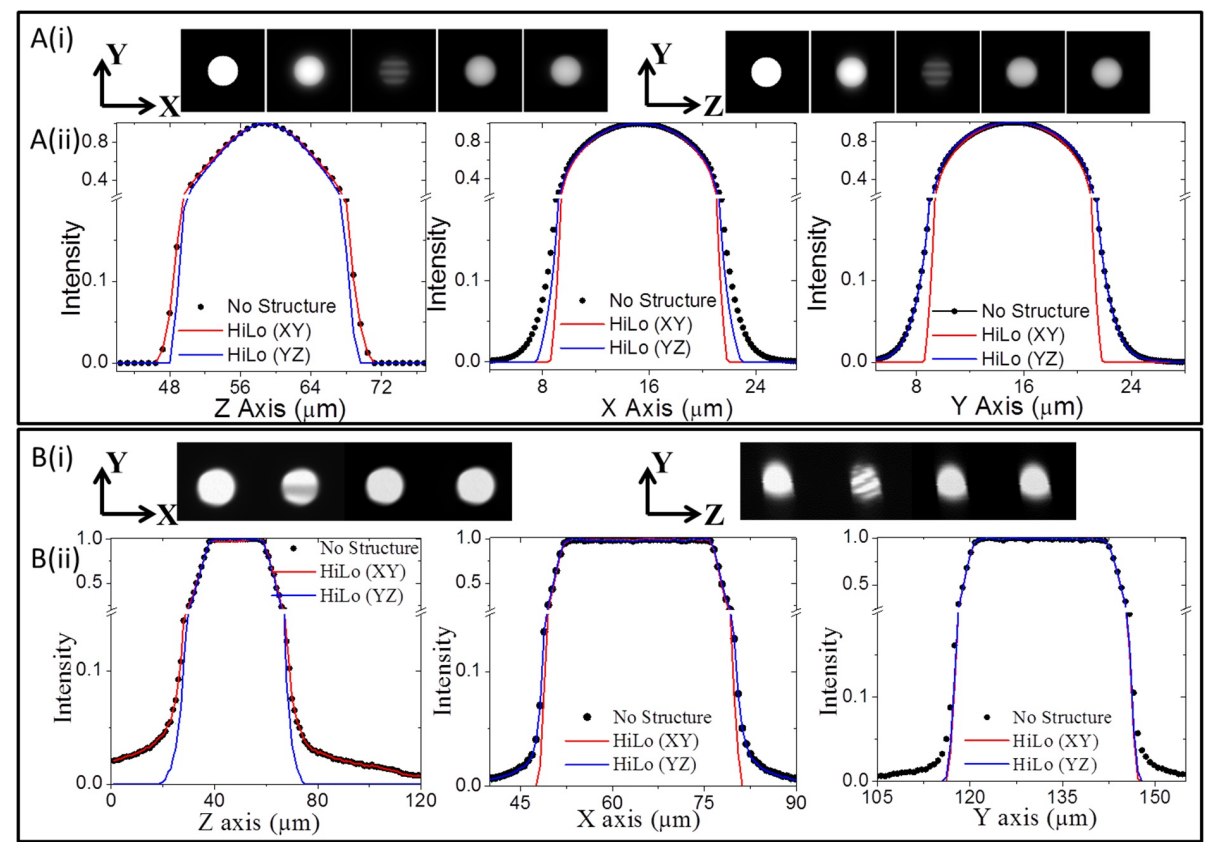

Fig. 3: Quantitative bead imaging to understand result of uniformly illuminated imaging, 3Phase imaging, conventional HiLo imaging and Combined axial and lateral Hilo imaging. (ai) Simulated bead images-object, uniformly illuminated images, grid projected images, conventional HiLo and combined lateral and axial HiLo image (XY and XZ projected images respectively). (a-ii) Line profiles along $Z, X$ and $X$ directions of normal images, conventional HiLo images, axial HiLo images, and combined HiLo images respectively. (b-i) Experimental bead images for uniformly illuminated images, grid projected images, conventional HiLo and combined HiLo image (XY and XZ projected images respectively). (b-ii) Line profiles along Z, $\mathrm{X}$ and $\mathrm{X}$ directions of experimental normal images, conventional HiLo images, axial HiLo images, and combined HiLo images respectively.

The simulation results are shown in Fig. 3(a). We find that the XY-HiLo image is closest to the original bead for both the lateral $\mathrm{X}$ and $\mathrm{Y}$ directions, but it does not yield any improvement along the $Z$ direction. Similarly, YZ-HiLo yields significant improvement in the $\mathrm{Z}$ and $\mathrm{Y}$ profiles of the bead, but not in $\mathrm{X}$. These results are intuitively satisfying and justify our motivation for combining the XY-HiLo and YZ-HiLo raw images into the final 3D HiLo image.

Figure 3(b) shows experimental data from a collection of $25 \mu \mathrm{m}$ size fluorescent beads excited at $488 \mathrm{~nm}$ and emitting at 530nm with other conditions matching those of the simulations. Qualitatively, the experimental data clearly agree with the simulations of Fig. 3(a), as XY-HiLo processing shows clear improvement in $\mathrm{X}$ and $\mathrm{Y}$ axial profiles of the image, whereas $\mathrm{YZ}$-HiLo shows improvement of $\mathrm{Y}$ and $\mathrm{Z}$ axis profiles. 


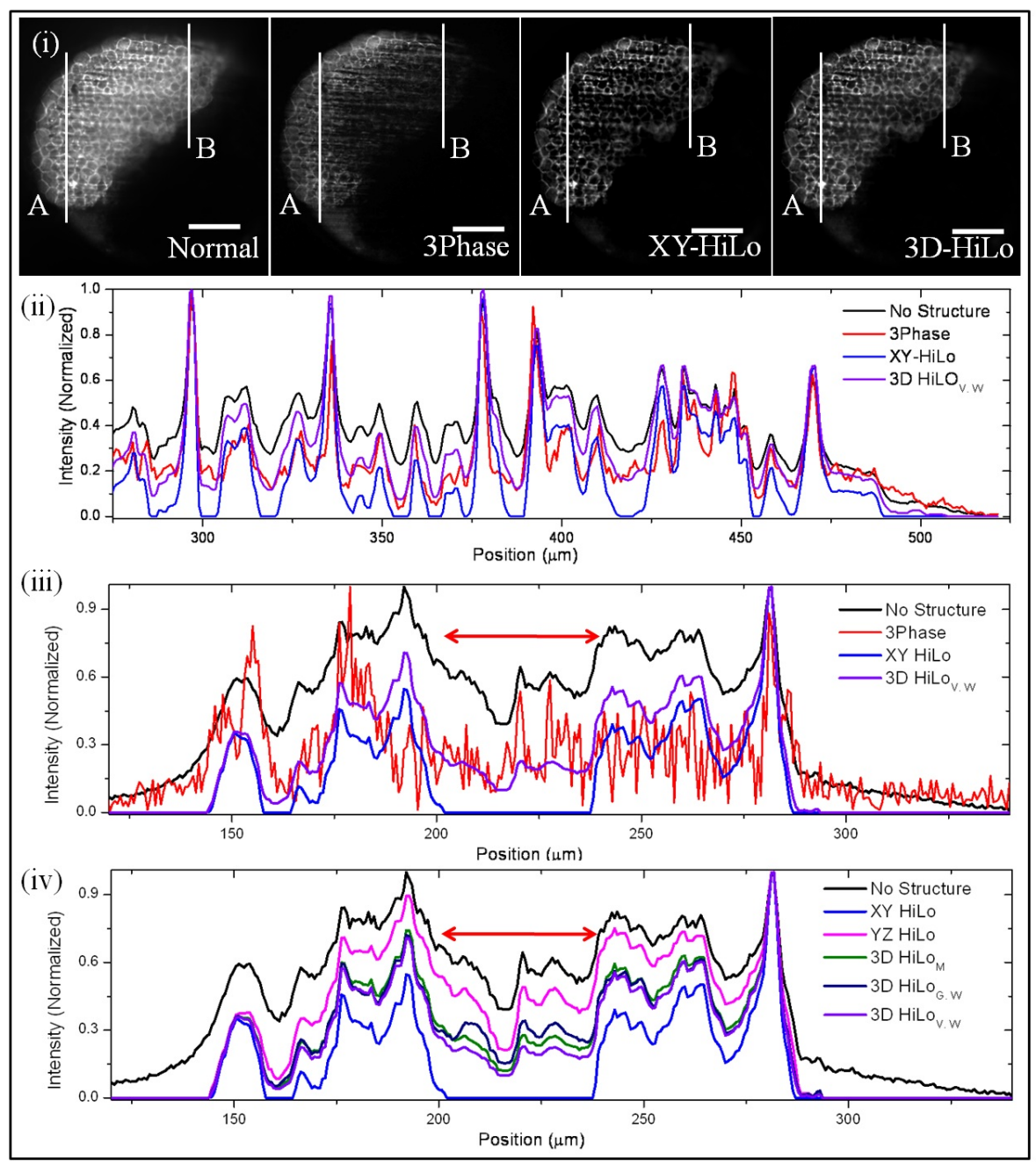

Fig.4: Images of the same membrane of zebrafish embryo (EGFP) at $30 \mu \mathrm{m}$ depth acquired with uniform illumination as well as different programs of structured illumination. The excitation is incident from the left direction in all experiments. (i) Normalized images of (from left to right): conventional (uniform illuminated) DSLM, 3Phase, conventional XY-HiLo and 3D HiLo (in this and ii, iii, we used the Variance Weighted method for 3D HiLo). (ii) Normalized intensity cross-sections of the images from line A (near the excitation) in Fig. 4(i). (iii) Normalized intensity cross-sections of the images from line B (far from the excitation) in Fig. 4(i). (iv) Comparison of the orthogonal HiLo images (XY, YZ) with conventional DSLM and the different methods described in Section 3 to combine the XY and YZ HiLo to obtain the 3D HiLo image.

To compare the quantitative effect of these image processing methods at different imaging depths, we imaged membrane-labeled Zebrafish-embryos imaged at $30 \mu \mathrm{m}$ depth using uniform illumination, 3Phase structured illumination, conventional XY-HiLo, and 3D HiLo post-processing. The results are shown in Fig. 4 as both reconstructed images and intensity cross-sections. The latter were obtained from two different regions, near (line A) and far (line B) from the laser excitation direction. We observe that 3Phase image removes most fluorescent features, except in the extreme left position, which corresponds to the lowest laser penetration and hence best grid contrast. On the other hand, conventional HiLo (equivalent to $\mathrm{XY}-\mathrm{HiLo}$ ) is much better at preserving features at lower laser penetration [line A, Fig. 4(ii)] 
whereas deeper inside the tissue many object structures are removed [line B, Fig. 4(iii)]. 3D HiLo does a much better job at recovering the deep sample structure [also line B, Fig. 4(iii)].

Another important observation is derived from the region denoted with a red arrow in Fig. 4(iii-iv). Conventional HiLo (XY-HiLo) removes valid low-bandwidth features of the object (also visible by conventional DSLM) because it low-pass filters them out. In 3D HiLo, the orthogonal (YZ) image picks them up because it does not low pass filter on the XY plane. This is the main benefit of using two orthogonally processed images. 3Phase also performs poorly in that region because it employs a subtraction that removes useful features and amplifies noise when the grid contrast is poor.

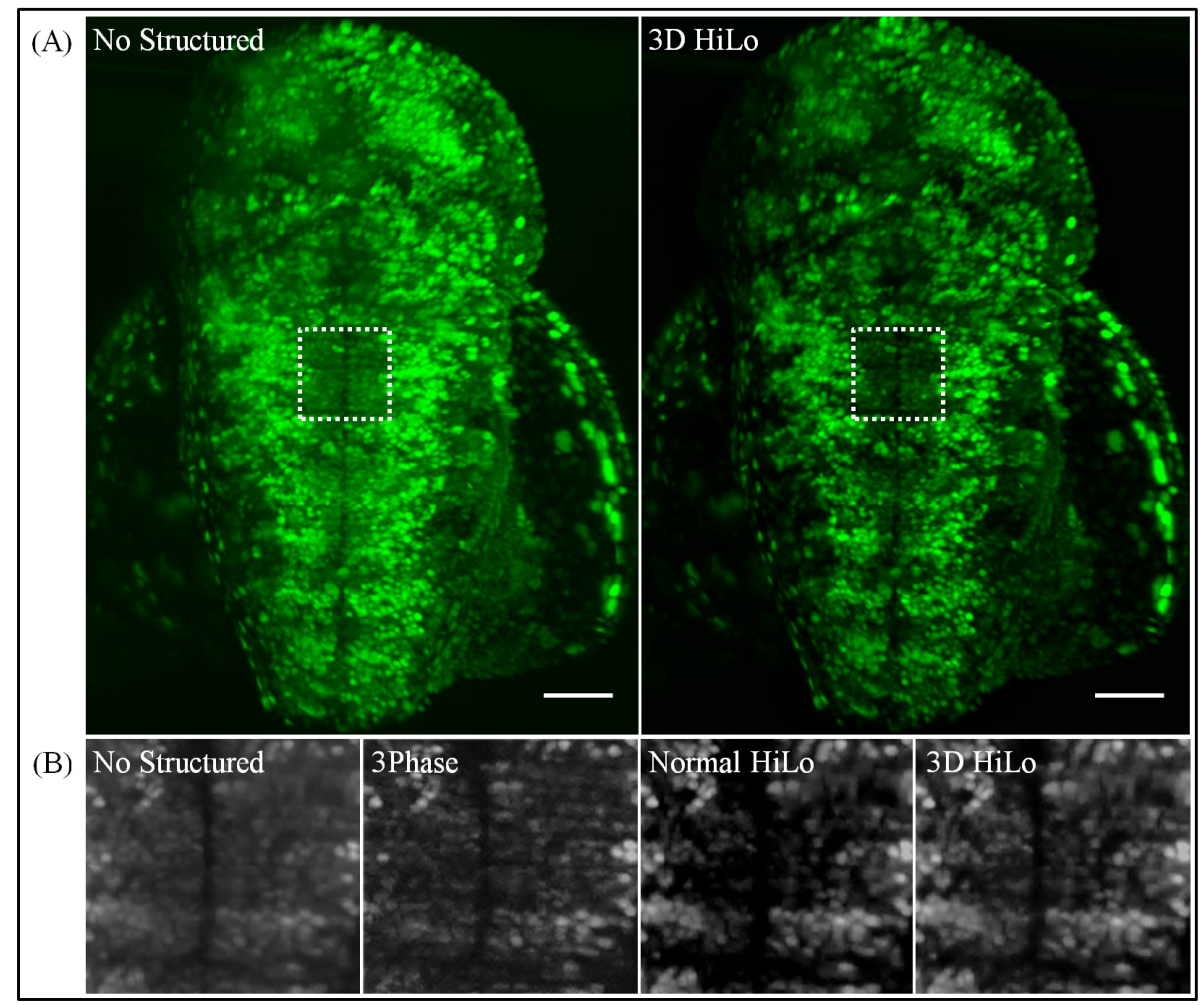

Fig. 5(a): Imaging spine structure of larval zebrafish using both uniformly illuminated images and 3D HiLo method (Variance Weighted). Here nuclei are tagged with EGFP. (b) Zoom in to the region showed as dotted line in (a), from a deeper fluorescent plane $(Z=110 \mu \mathrm{m})$, for uniformly illuminated, 3Phase, conventional HiLo and combined HiLo methods, from left to right, respectively. Scale bar $=100 \mu \mathrm{m}$.

The significance of grid contrast variation and recovery of corresponding object figures in the two orthogonal planes XY and YZ is illustrated in Fig. 4(iv). Confirming our intuition about the different ways of combining the two orthogonally processed methods, we can see that the Variance Weighted 3D HiLo provides a better contrast enhancement than Mean 3D HiLo, Geometrically Weighted 3D HiLo.

Figure 5 shows images acquired in the case of a representative difficult $3 \mathrm{D}$ imaging problem, the spine-structure of $36 \mathrm{hpf}$ (hours post fertilization) larval zebrafish. These images were composed from stacking the $3 \mathrm{D}$ reconstructions from the respective methods and then performing a standard Maximum Intensity Projection (MIP) operation. At a deep fluorescent plane $(Z=110 \mu \mathrm{m})$, images post-processed with the different techniques clearly show that $3 \mathrm{D}$ HiLo can remove background in a thick tissue sample without removing any features of the 
object, whereas both XY HiLo and 3Phase-image remove some object features along with background noise.

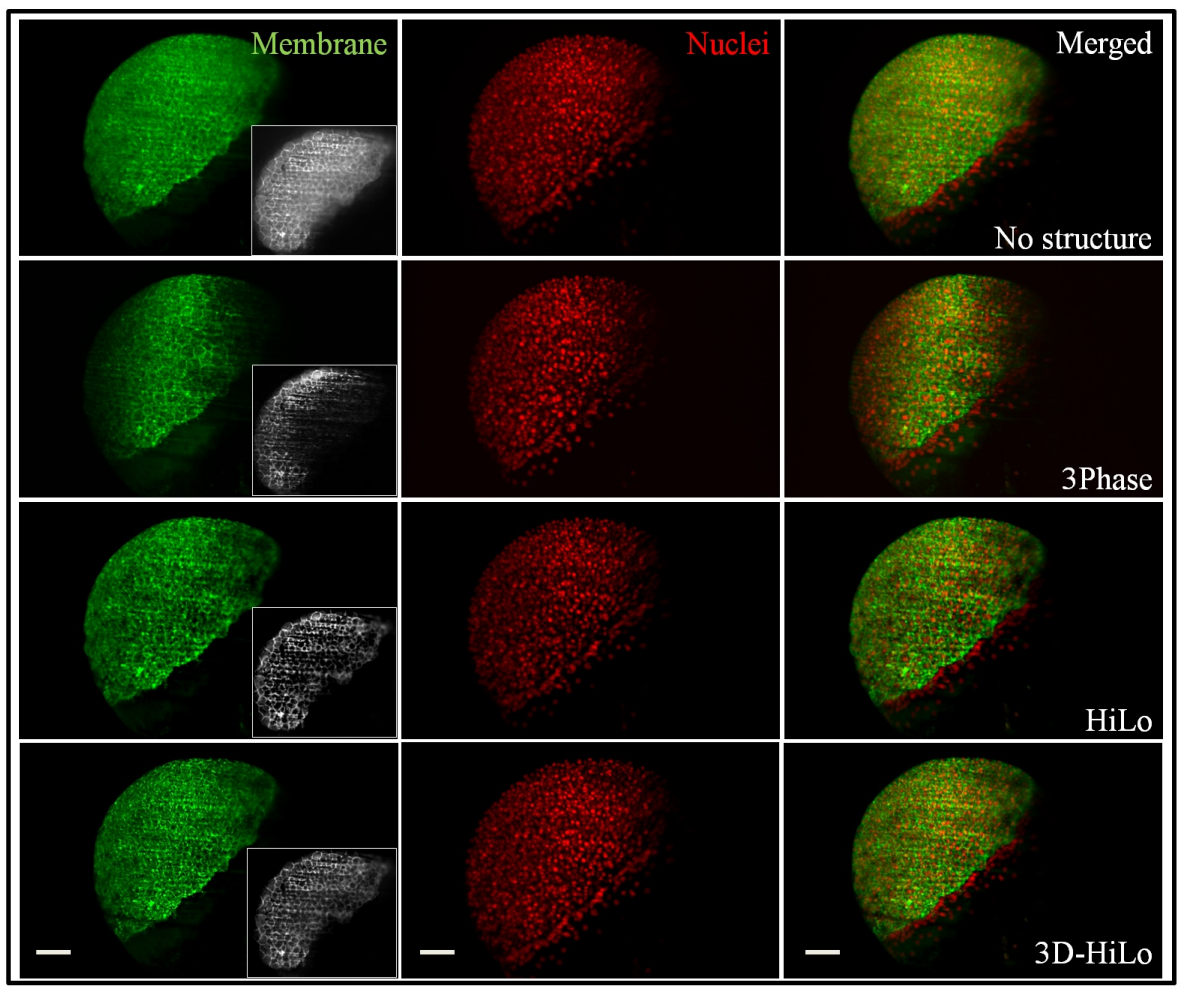

Fig. 6: Zebrafish embryo images acquired and post-processed with different programs of structured illumination. Nuclei are stained with Histone H2B-mRFP (RED) and Cell membranes are stained with membrane-EGFP (pCAG-mGFP-GREEN). Inset: Membrane images of single fluorescent planes at $30 \mu \mathrm{m}$ depth image of the sample for corresponding images. Merged images are shown in right most columns. (Scale bar $=100 \mu \mathrm{m}$ ).

Finally, Fig. 6 shows 3D images of zebrafish embryo (6 hpf) post-processed with the same suite of techniques as in Fig. 5 and displayed using MIP. This specimen contains both heterogeneous low-frequency structures (membranes) and discrete high-frequency objects (nuclei), which have been labeled with different fluorophores so they are observable separately. We observe that for high frequency objects the improvement of either 3Phase or HiLo over uniform (unstructured) DSLM illumination is minimal. However, 3D HiLo significantly improves the image quality of the membranes.

In addition to the obvious one of requiring two raw images instead of three, we have observed certain additional advantages of HiLo over 3Phase for thick-large tissue imaging. As 3Phase is a subtraction based method, its effectiveness is strongly contingent upon grid contrast. Therefore, at image portions where grid contrast is low, or image portions where the fluorescent signal is lower, 3Phase fails dramatically. In practice, such failures are common for thicker and deeper portions of the tissue. Moreover, the 3Phase method is very sensitive on the relative phase of the three raw images. Slight deviation from theoretical phase in the image can result in major image processing artifacts, e.g. line patterns in the post-processed image.

Even though we took great effort to minimize experimental errors in the relative phases, contrast-related artifacts are vividly displayed in the 3Phase result for the membrane in Fig. 6, where the left-hand side of the object appears darker even though the excitation there was stronger and the illumination grid contrast should be higher at that side of the object (recall 
that the excitation illumination is always coming from the left in our experimental results). The reason for the darker appearance on the left-hand side is that the fluorophore concentration was lower in that region. We also have found few severe line pattern artifacts due to phase mismatch, especially in deeper tissue portions. Hence, we conclude that 3 Phase structured illumination works well for thinner and more transparent tissue samples, where one can imprint good-contrast structured imaging throughout the volume and the phase shift is precise; whereas for thicker samples, where grid contrast is liable to vary widely across the 3D sample size, 3D HiLo is preferable.

\section{Discussion}

In summary, we have developed a novel digital scanned laser sheet microscope capable of capturing raw images with different programs of structured illumination and post-processing them according to algorithms optimized for the corresponding caveats of illumination grid contrast decay and scattering artifacts inside the sample. We have proposed a method of combining orthogonally processed HiLo images from two planes: one oriented as in conventional DSLM imaging (XY) and one perpendicular to it (YZ). We have shown quantitatively that 3D HiLo provides better image contrast than both conventional HiLo and 3Phase, without removing significant object features together with the background. We have also compared the different techniques in terms of local sample structure, e.g. discrete spatially confined objects such as nuclei versus heterogeneous extended low-frequency objects such as membranes.

Much work remains to be done in optimizing the programs of illumination. Here we presented only a simple sinusoidal raster scan, whereas obviously one could try alternate schemes of illumination, especially if object priors can be taken into account to design illumination programs tailored to the specific object or object class. Moreover, our simple linear convex method of combining the orthogonal HiLo images XY and $\mathrm{YZ}$ into a single 3D HiLo image can be extended to more sophisticated nonlinear or iterative methods, also taking object priors into account.

\section{Acknowledgments}

This research was supported by the National Research Foundation, Singapore through the Singapore MIT Alliance for Research and Technology's BioSystems and Micromechanics Inter-Disciplinary Research programme (015824-039) and Center for BioImaging Sciences, NUS (R-154-000-413-720), and the U.S. National Institutes of Health through grant (1R01CA134424-01A1).

\#176294 - \$15.00 USD Received 14 Sep 2012; revised 25 Oct 2012; accepted 26 Oct 2012; published 20 Nov 2012

(C) 2012 OSA

3 December 2012 / Vol. 20, No. 25 / OPTICS EXPRESS 27347 